\title{
Design, Realization and Implementation of a Novel Phantom for Quality Control of Micro-SPECT/CT and Micro-PET/CT Systems
}

\author{
Hatem Besbes $^{1 *}$, Radhia Besbes Krid ${ }^{2}$, Bassel Solaiman ${ }^{3}$ \\ ${ }^{1}$ Laboratory of Biophysics and Medical Technology, Faculty of Medicine, Tunis El Manar University, Tunis, Tunisia \\ ${ }^{2}$ Department of English, Languages Institute of Gabes, Gabes University, Gabes, Tunisia \\ ${ }^{3}$ Department of Imagery and Information Processing, Telecom Bretagne, Brest, France \\ Email: besbesh@gmail.com
}

Received 28 December 2014; accepted 12 January 2015; published 19 January 2015

Copyright (C) 2015 by authors and Scientific Research Publishing Inc.

This work is licensed under the Creative Commons Attribution International License (CC BY).

http://creativecommons.org/licenses/by/4.0/

CC) (i) Open Access

\begin{abstract}
The proposed phantom is designed for the quality control of micro-SPECT/CT and micro-PET/CT systems. However, it is an assembly of six patterns stored in a cylindrical box enabling to control both micro-SPECT unit in terms of uniformity, spatial linearity and spatial resolution and micro-CT unit in terms of uniformity, spatial linearity, spatial resolution, diffusion rate, low contrast detectability, Hounsfield unit linearity and slice thickness. The construction material is Plexiglas. As for the implementation, it was made on a micro-SPECT/CT machine of the type "speCZT eXplore CT 120". Compared to the NEMA NU 4-2008 image quality phantom, this phantom offers micro-CT quality control and is more efficient in control of spatial resolution for micro-SPECT and microPET systems.
\end{abstract}

\section{Keywords}

Phantom, Quality Control, Micro-SPECT/CT, Micro-PET/CT

\section{Introduction}

Recently, preclinical imaging has evolved considerably but the evolution has been only concerned with small animals (mice, rats...). Although the results obtained in preclinical tests are extremely useful in the fields of

${ }^{*}$ Corresponding author.

How to cite this paper: Besbes, H., Krid, R.B. and Solaiman, B. (2015) Design, Realization and Implementation of a Novel Phantom for Quality Control of Micro-SPECT/CT and Micro-PET/CT Systems. J. Biomedical Science and Engineering, 8, 46-55. http://dx.doi.org/10.4236/ibise.2015.81005 
clinical imaging [1]-[4] and pharmacology [5] [6], given the size of these animals, the instruments used have imaging performance well above those used for humans.

The expected results of the obtained images are so important that we must be quite demanding when regarding their qualities. The continual production of the best qualities of these images is ensured by the development of adequate quality control protocols [1] [7] [8]. These protocols are developed in relation to quality control protocols already adopted in clinical imaging.

As in clinical imaging, quality control in preclinical imaging is usually done through the acquisitions of images on specific phantoms [9] [10]. These phantoms are generally formed by one or more compartments, each of which simulates a situation to evaluate one or more quality parameters of an explored preclinical imaging system. In the present state of things, it is a combination of two imaging systems providing two different types of images. Indeed, it is a micro-nuclear imaging system (micro-SPECT or micro-PET) combined with a micro-computed tomographic imaging system (micro-CT). In practice, only the quality of a micro-nuclear imaging unit is considered, which is a bit inconsistent as long as the micro-SPECT and micro-PET slices are located through micro-CT acquisitions. In clinical imaging (SPECT or PET and CT), the two corresponding systems are controlled independently of each other with two different phantoms. The proposed phantom allows the quality control of both micro-nuclear imaging and micro-CT systems with a single acquisition protocol. It contains patterns that control the same quality parameters of both machines as the uniformity, spatial linearity and spatial resolution, and other additional patterns for specially quality control of the micro-CT unit as the diffusion profile, Hounsfield unit linearity, the low contrast detectability and slice thickness. The material of construction selected for the phantom realisation is Plexiglas.

\section{Materials and Methods}

\subsection{Design}

For the design of the phantom, the SOLIDWORKS software is considered for reasons of flexibility and easiness of conception.

\subsection{Construction Materials}

For the realization of the phantom, the material used is Plexiglas, which is easy to machine and is cheaper than and as efficient as plastic acrylate. For fixing the structure, polyamide screws are used.

\subsection{Quality Parameters}

Knowing that, like SPECT/CT and PET/CT machines, both micro-SPECT/CT and micro-PET/CT systems are each one composed by two units (micro-nuclear imaging unit and micro-CT unit), quality control operations are performed individually on each unit. Certain quality control protocols don't need image acquisitions on phantoms like visual inspection, measurement of the sensitivity for micro-SPECT or micro-PET and light alignment and measurement of the dose at skin entrance (DSE) for micro-CT. However, the majority of quality control protocols need image acquisitions on specific phantoms. For micro-nuclear imaging unit, quality control protocols concern uniformity, spatial linearity, spatial resolution, pixel size and centre of rotation (COR) [11] [12]. While for micro-CT unit, quality control protocols concern uniformity, noise, low contrast detectability, spatial linearity (geometric distortion), slice thickness, diffusion profile, spatial resolution, and Hounsfield unit linearity [12]-[14]. Moreover, Hounsfield unit for a substance is given by Equation (1) [14].

$$
\mathrm{HU}=\frac{\mu_{\text {substance }}-\mu_{\text {water }}}{\mu_{\text {water }}} \cdot 1000
$$

where $\mu_{\text {substance }}$ is the resultant $\mathrm{X}$-ray attenuation coefficient for the substance, $\mu_{\text {water }}$ is the $\mathrm{X}$-ray attenuation coefficient for water.

\subsection{Image Acquisitions}

For the implementation of the considered phantom, acquisitions are conducted on micro-SPECT/CT machine of the type "speCZT eXplore CT 120" (GE Healthcare) (Figure 1) of the in vivo preclinical imaging service in the 


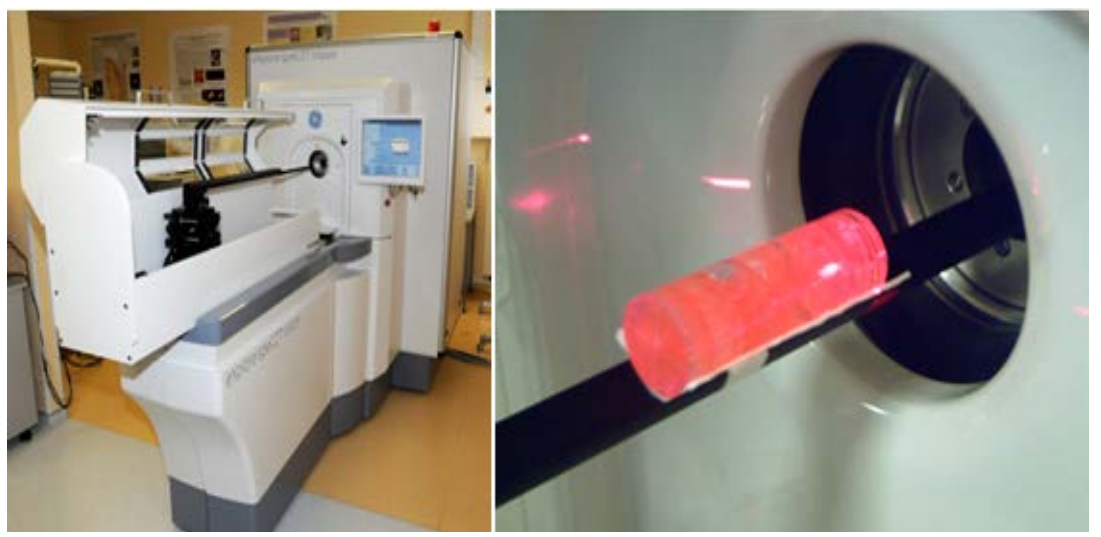

Figure 1. Phantom placed in the tunnel of the speCZT eXplore CT 120 machine.

Hospital Hautepierre of Strasbourg. This camera is composed of a micro-SPECT unit and micro-CT unit. The detection head of the micro-SPECT unit "eXplore speCZT" consists of ten sensors plan CZT semiconductor with a surface of $64 \mathrm{~cm}^{2}$ and $5 \mathrm{~mm}$ thick. The detectors are stationary and located in the form of a decagon around a rotating and interchangeable cylindrical collimator [1] [2] [5]. For the CT unit "Explore CT 120", it is constituted by an X-ray high efficiency tube of $5 \mathrm{~kW}$ whose voltage can vary between 70 and $120 \mathrm{kV}$ and is attached to a planar array of CCD detectors $3500 \times 2300$. The X-ray beam used for the micro-CT unit is conical [2]. Acquisitions can also be done on a micro-PET/CT system with the same protocol because micro-PET and micro-SPECT are both nuclear tomographic imaging techniques with low differences between their principle quality parameters [16].

\section{Results and Discussion}

\subsection{Design of the Phantom}

When designing the proposed phantom (Figure 2), the major considered constraints are the possibilities of verifying the majority of acquisition parameters qualities for micro-SPECT/CT and micro-PET/CT systems while respecting the dimensions of acquisition areas offered by each machine. For this, it is better to refer to the NEMA accreditation in case of micro-SPECT and micro-PET [17] and ACR accreditation in case of micro-CT [18]. Seven quality control parameters have been fixed: uniformity (micro-SPECT or micro-PET and micro-CT units), spatial linearity (micro-SPECT or micro-PET and micro-CT units), spatial resolution (micro-SPECT or micro-PET and micro-CT units), diffusion profile (micro-CT unit), Hounsfield unit linearity (micro-CT unit), low contrast detectability (micro-CT unit) and slice thicknesses (micro-CT unit). With the exception of uniformity, which can be measured following an acquisition done on the homogeneous solution, for each of the selected parameters, we designed a pattern that enables us to evaluate it (Figure 2). The patterns are stored in a cylinder (Part 1) with sealing cover (Parts 8, 9, 10, 11 and 12), which constitute the body of the obtained phantom. Whereas, the spatial linearity pattern has a linear geometry in both directions $\mathrm{x}$ and $\mathrm{y}$ of the plan (Part 2), the spatial resolution pattern for micro-SPECT or micro-PET units contains variable diameter cylindrical hole pairs (Part 3), the diffusion profile pattern is made of a thin metal wire (Part 4), the spatial resolution pattern of micro-CT is a thin prismatic needle (Part 4), the Hounsfield unit linearity pattern contain the same volume of materials having Hounsfield units describing the Hounsfield scale from -1000 for air to +1000 for bone (Part 5), the low contrast detectability pattern is made by two thin plates with different thickness in which are perforated the same set of variable diameter holes (Part 6) and the slice thickness pattern is made essentially of two thin opposite metal ramps with the same inclination angle (Part 7).

\subsection{Realization of the Phantom}

Following the fixed design and using Plexiglas cylinders with a diameter of $3 \mathrm{~mm}$, the proposed phantom is realized (Figure 3). It is composed of the following:

- The phantom body that is a cylindrical enclosure with a sealed cover and two holes for the introduction of 


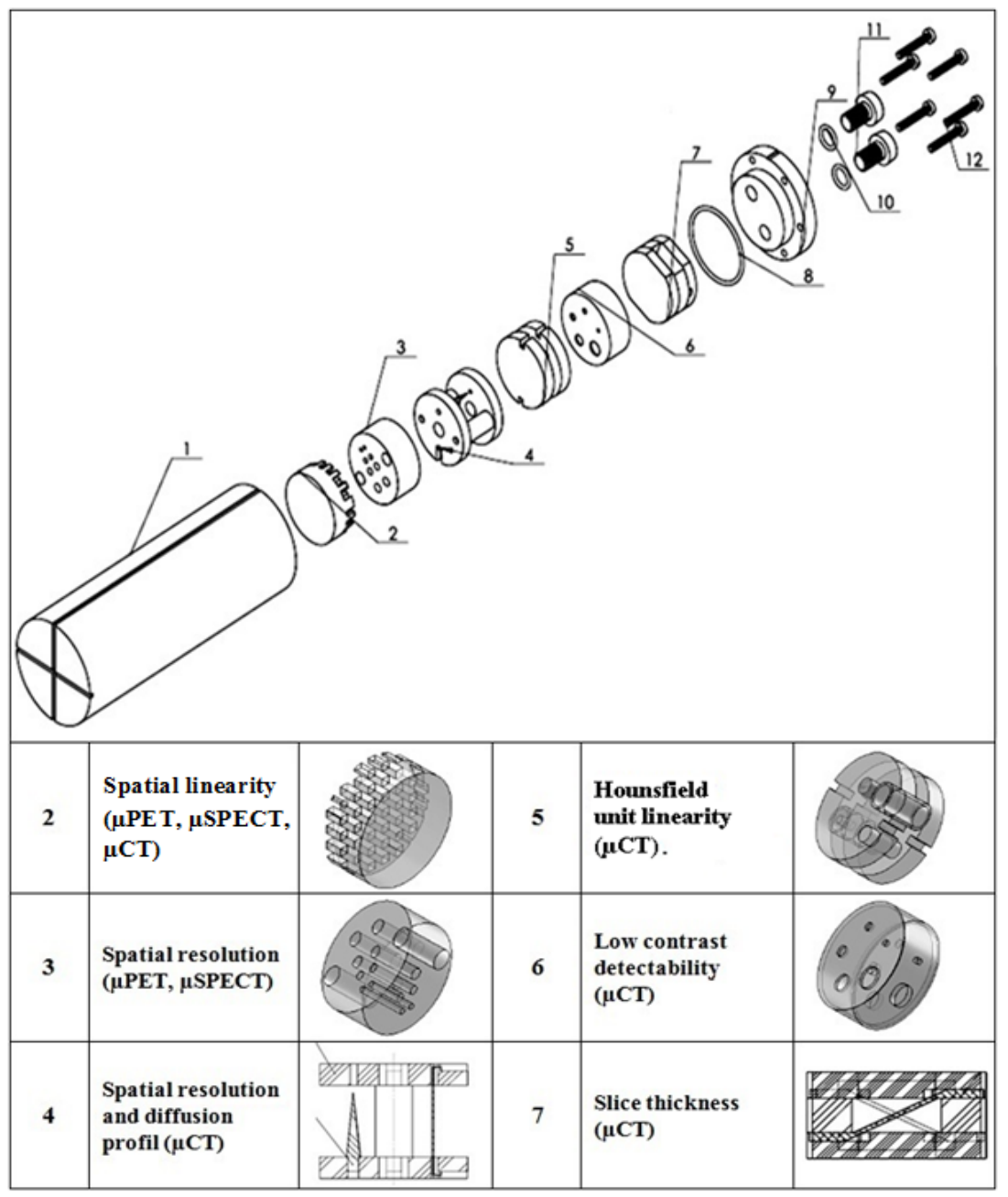

Figure 2. Design of the phantom with SOLIDWORKS.

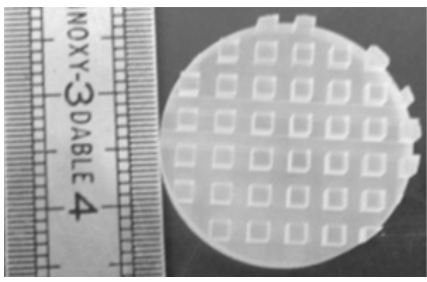

(a)

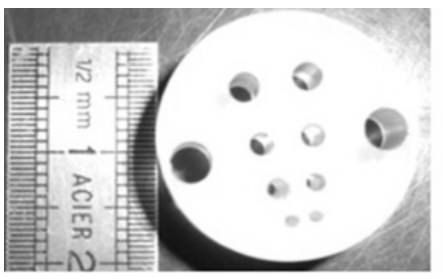

(d)

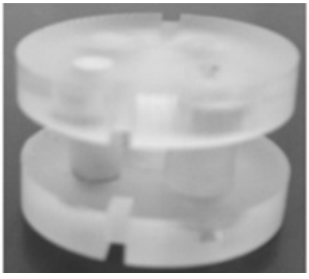

(b)

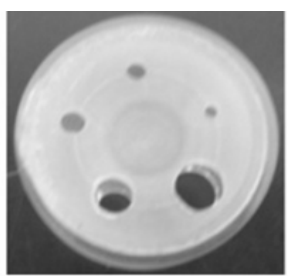

(e)

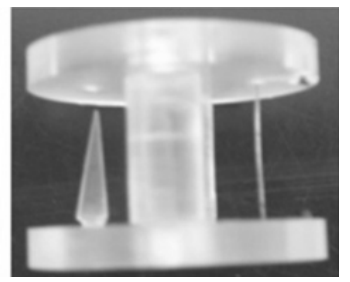

(c)

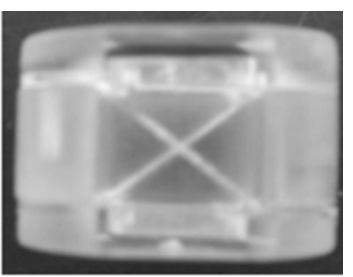

(f)

Figure 3. Patterns contained in the phantom (a) Spatial linearity (micro-PET, microSPECT, micro-CT); (b) Hounsfield unit linearity (micro-CT); (c) Spatial resolution and diffusion profile (micro-CT); (d) Spatial resolution ( $\mu$ PET, micro-SPECT); (e) Low contrast detectability (micro-CT); (f) Slice thickness (micro-CT). 
solutions with contrast agents (Figure 4). The patterns for control of various performances are housed in the cylinder.

- The pattern of spatial linearity, which is in the form of a solid cylinder in which we have made orthogonal and periodic grooves $1.5 \mathrm{~mm}$ wide, $3 \mathrm{~mm}$ deep and spaced $1.5 \mathrm{~mm}$ (Figure 3(a)).

- The pattern of Hounsfield unit linearity that consists of four cylinders of the same size and which Hounsfield units are indexed by the manufacturer: the first is filled with water $(\sim 0 \mathrm{HU})$, the second is filled with polyethylene ( $\sim 80 \mathrm{HU})$, the third is filled with teflon $(\sim+1000 \mathrm{HU})$ and the fourth is filled with air ( $\sim 1000 \mathrm{HU})$ (Figure 3(b)).

- The pattern of spatial resolution and the diffusion profile for micro-CT that contains a copper wire $0.2 \mathrm{~mm}$ thick elongated parallel to the axis of the phantom and a prismatic needle with a square base $\left(2.5 \times 2.5 \mathrm{~mm}^{2}\right)$ (Figure 3(c)).

- The pattern of spatial resolution of micro-SPECT and micro-PET shows as a solid cylinder in which we operated four pairs of holes with diameters of 1, 1.5, 2 and $2.5 \mathrm{~mm}$ (Figure 3(d)).

- The pattern of low contrast detectability that is made of two disks 0.5 and $1 \mathrm{~mm}$ thickness in which are pierced in the same position five holes of diameters 1, 1.5, 2, 3 and $4 \mathrm{~mm}$ (Figure 3(e)).

- The pattern of slice thickness measurement in micro-CT that consists of two opposite ramps of aluminium with a thickness of $0.6 \mathrm{~mm}$ and an inclination angle $\theta$ of $25.36^{\circ}$ (Figure 3(f)).

\subsection{Acquisitions}

After the realization phase, begins the acquisition phase. For this, the phantom is filled with a radioactive solution $\left(\mathrm{V}=11.5 \mathrm{~cm}^{3}\right)$ made of demineralised and degasified (for 48 hours) water +1 drop of aqueous eosin $2 \%$ (red colour of solution) $+0.1 \mathrm{ml}$ of pertechnetate-99 m solution (activity $=4.10 \mathrm{mCi})+1$ drop of Iomeron 400 (contrast agent for X-ray imaging). After filling, the considered phantom is placed in the tunnel of the machine (Figure 1), then the acquisition parameters are set (Table 1) and acquisition protocol is started.

At the end of the acquisition protocol, three types of images are obtained: micro-CT, micro-SPECT and microSPECT/CT.

The micro-CT images are obtained through transversal acquisitions at the levels of the homogeneous region of the proposed phantom and patterns number 2, 4, 5, 6 and 7 (Figure 2). They concerned uniformity (Figure 5(a)), spatial linearity (Figure 5(b)), Hounsfield unit linearity (Figure 5(c)), low contrast detectability (Figure 5(d)), diffusion profile (Figure 5(e)) and slice thickness (Figure 5(f)).

Uniformity U (Figure 5(a)) is calculated knowing the mean value of Hounsfield peripheral numbers (HNP) and the Hounsfield central number $\left(\mathrm{HN}_{\mathrm{C}}\right)$ for the water (without contrast agent) (Equation (2)) [14].

$$
\mathrm{U}=\frac{\mathrm{HN}_{\mathrm{P}}-\mathrm{HN}_{\mathrm{C}}}{\mathrm{HN}_{\text {Water }}-\mathrm{HN}_{\text {Air }}} \cdot 100 \%
$$

For the spatial linearity or geometric distortion (GD) test (Figure 5(b)), it is matter of comparing the measured dimensions to the real dimensions in the two direction $\mathrm{x}, \mathrm{y}$ of the plan (Equation (3)).

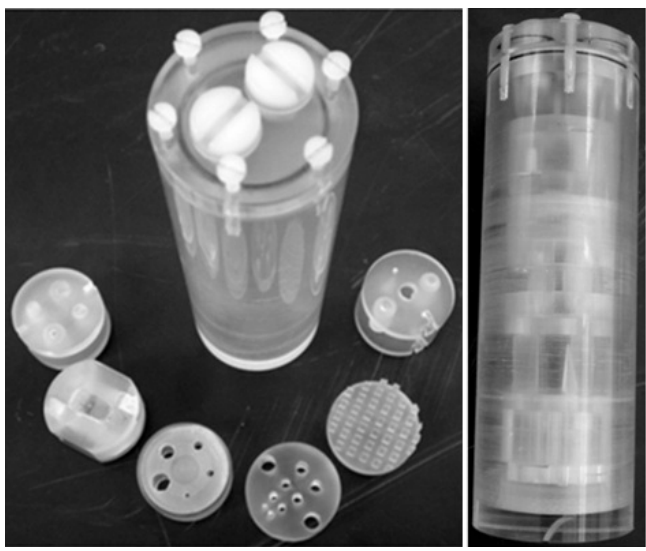

Figure 4. The phantom. 
Table 1. Acquisition parameters.

\begin{tabular}{cl}
\hline \multicolumn{1}{c}{ Micro-CT } & \multicolumn{1}{c}{ Micro-SPECT } \\
\hline & Activity: $2.8 \mathrm{mCi}$ \\
& Collimator: 10 pinholes (mise) \\
Acquisition mode: Static & Acquisition mode: Static \\
Acquisition time: $45 \mathrm{~s}$ & Acquisition time: $1 \mathrm{~h} 28 \mathrm{mn}$ \\
& Energy window: $125 \mathrm{keV}$ à $150 \mathrm{keV}$ \\
& Count rate: $586 \mathrm{cps} / \mathrm{s}$ \\
& Total count: $16348.116 \mathrm{~K}$ \\
\hline
\end{tabular}
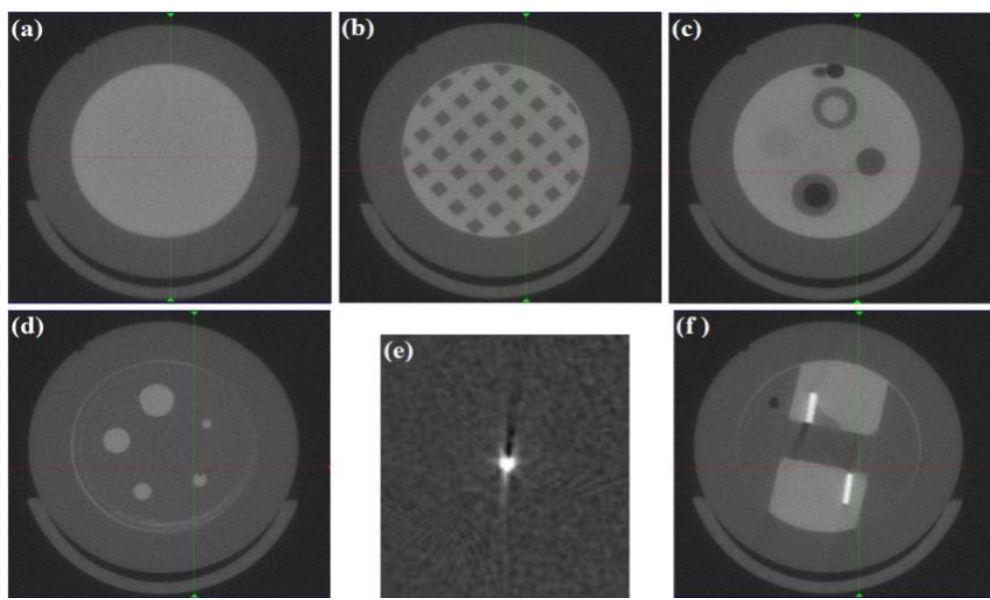

Figure 5. Micro-CT acquisitions ((a) Uniformity, (b) Spatial linearity, (c) Hounsfield unit linearity, (d) Low contrast detectability, (e) Diffusion profile, (f) Slice thickness).

$$
\mathrm{GD}_{\mathrm{x}, \mathrm{y}}=\left|\frac{\text { measured dimension }- \text { real dimension }}{\text { real dimension }}\right| \times 100
$$

Diffusion profile (DP) is defined as the comparison between the measured diameter on the image and the real diameter of the tin cooper wire (Equation (4)).

$$
\mathrm{DP}=\frac{\text { measured diameter }- \text { real dimeter }}{\text { real diameter }} \times 100
$$

For the remaining parameters, although affected by the presence of the contrast agent (Iomeron 400), spatial resolution and low contrast detectability are measured as the smallest detail that can be distinguished on the appropriate pattern image, the slice thickness (ST) measurement is done when knowing the foul width at half maximum (FWHM) of the orthogonal profile at the level of aluminium ramps (Figure 5(f), Figure 6), and the Hounsfield units for each material are displayed by the micro-CT system.

The micro-SPECT images are obtained through transversal acquisitions at the levels of the homogeneous region of the proposed phantom and patterns number 2 and 3 (Figure 2). They concerned uniformity (Figure 7(a)), spatial resolution (Figure 7(b)) and spatial linearity (Figure 7(c)).

For micro-nuclear imaging (micro-SPECT and micro-PET), as for nuclear imaging, there are two types of uniformity; integral uniformity (UI) and differential uniformity (UD). Integral uniformity is calculated knowing the maximum and minimum pixel count values (C) in homogeneous solution (Equation (5)), while differential uniformity depends on the maximum and minimum pixel count values every set of 5 contiguous pixels in rows or columns (Equation (6)) [11]. Spatial resolution is evaluated, as for micro-CT, as the smallest detail that can be 


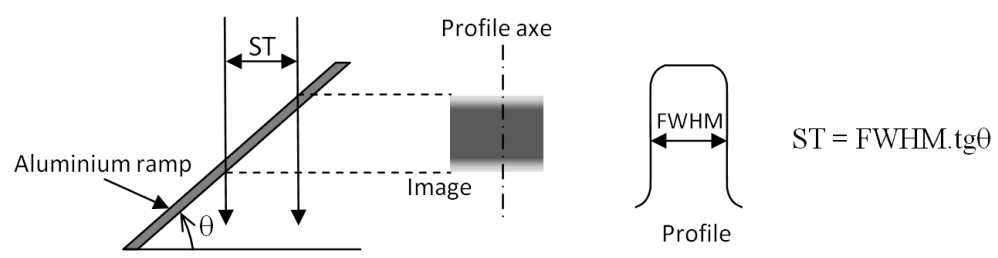

Figure 6. Slice thickness calculation.
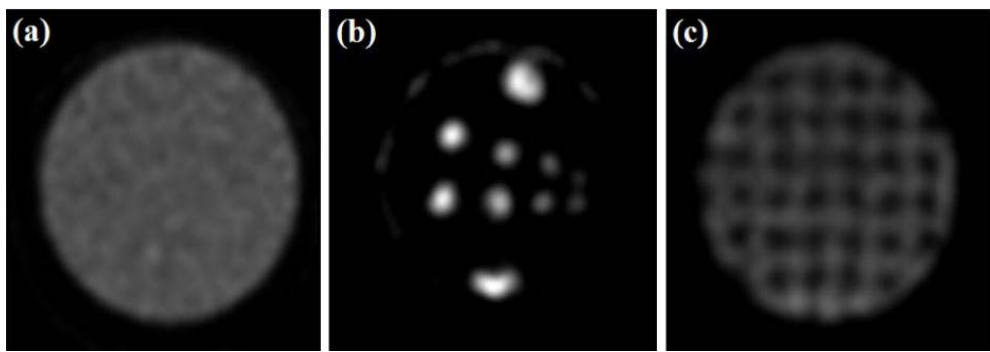

Figure 7. Micro-SPECT acquisitions ((a) Uniformity, (b) Spatial resolution, (c) Spatial linearity).

distinguished on the image of the pattern number 3 (Figure 7(b)). The test of spatial linearity is done by visual analysis of the micro-SPECT acquisition on the pattern number 2 (Figure 7(c)).

$$
\begin{gathered}
\mathrm{UI}=\frac{\left(\mathrm{C}_{\max }-\mathrm{C}_{\min }\right)}{\left(\mathrm{C}_{\max }+\mathrm{C}_{\min }\right)} \times 100 \\
\mathrm{UD}=\operatorname{Max}\left[\frac{\left(\mathrm{C}_{\max }-\mathrm{C}_{\min }\right)}{\left(\mathrm{C}_{\max }+\mathrm{C}_{\min }\right)} \times 100\right]
\end{gathered}
$$

Micro-SPECT/CT acquisitions are made following the micro-CT acquisitions. Indeed, it comes to be positioned on the micro-CT acquisitions for achieving the micro-SPECT acquisitions, and then we proceed with image fusions of the two modalities for each slice. This operation concerned acquisitions made at the level of patterns number 2 and 3. Thus, we are able to test micro-SPECT/CT image of spatial linearity (Figure 8(a)) and spatial resolution (Figure 8(b)).

\subsection{Interpretation}

The proposed phantom allowed, simultaneously, the quality control of the micro-SPECT unit and that of the microCT unit and consequently of the whole micro-SPECT/CT system and the same thing for a micro-PET/CT system. This permit to do all acquisitions for quality tests with just one protocol.

For the micro-SPECT unit, it was found that the spatial resolution is close to $1 \mathrm{~mm}$ as it was possible to distinguish a boundary separation between the images of the two holes of $1 \mathrm{~mm}$ diameter and separated by a distance also of $1 \mathrm{~mm}$ (Figure 7(b)). In addition, the spatial linearity test reveals the presence of geometric distortions in the middle region of the useful field of view (Figure 7(c)) was remarked. So, it is necessary to do spatial linearity correction of the micro-SPECT system detector.

For the micro-CT acquisitions, the spatial linearity of this unit was tested (Figure 5(b)) and the diffusion profile was easy to measure on Figure 5(e) using Equation (4). As for uniformity, it was still easy to calculate on Figure 5(a). While the Hounsfield units of Figure 5(c) were clearly measurable for air, Teflon and polyethylene, they were not in the case of water because of the presence of the contrast agent (Iomeron 400). The same for the low contrast detectability (Figure 5(d)), because the Iomeron 400 increased shift of X-ray attenuation rate between the Plexiglas and the solution, which makes the condition of low water-Plexiglas contrast (Figure 9) no longer there. So, it is convenient to work without any contrast agent. Moreover, to measure slice thickness, low contrast with water gives more uncertain results (Figure 9). Thus, the results in this side are better with the contrast agents (Figure 5(f)). 

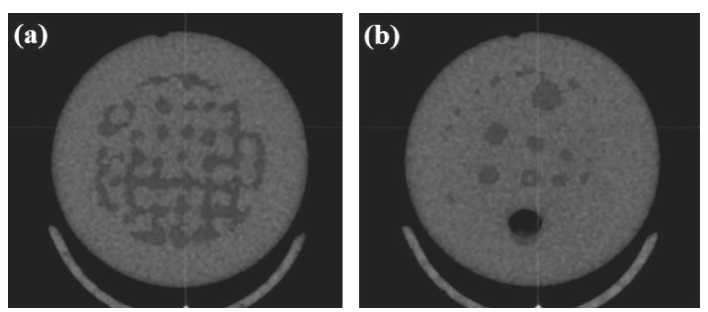

Figure 8. Micro-SPECT/CT acquisitions ((a) Spatial linearity, (b) Spatial resolution).

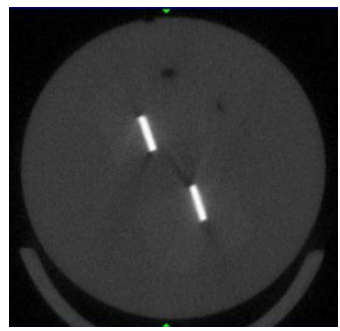

Figure 9. Image test for slice thickness in micro CT without Iomeron 400.

For micro-SPECT/CT acquisitions (fusion), it was noticed that the global uniformity parameter is rather imposed by micro-SPECT unit which presents weaker qualities compared to micro-CT unit. The same applies to geometric distortions; they are more pronounced in Figure 8(a). For spatial resolution of the whole system, it is better than $1 \mathrm{~mm}$ because the fusion with micro-CT can improve this quality. These constitute the advantages of multimodal imaging systems (micro-SPECT and micro-CT).

The use of Iomeron 400 is justified by the fact that it allows a better visualization of all details in the proposed phantom by increasing the contrast between the solution and the Plexiglas. This constitutes a better demonstration for users.

The phantom is also extremely flexible. Then it is possible to remove or to change the patterns according to the quality control situation.

\subsection{Comparison with the NEMA Phantom}

Since there are no hybrid phantoms for quality control of micro-SPECT/CT and micro-PET/CT systems, the NEMA NU 4-2008 image quality phantom was chosen for the comparison with the proposed phantom [10] [19] [20]. On the one hand, this allows saying that the proposed phantom is the first in the field of simultaneous quality control of micro-CT units and micro-SPECT or micro-PET units. Besides, the comparison is restricted to the quality control of micro-SPECT unit. For this, acquisitions with the NEMA phantom were realized (Figure 8) with the same preparation and acquisition conditions carried out for the proposed phantom (Table 1). Thus, the NEMA phantom allows only the controls of uniformity (Figure 10(a)), spatial resolution (Figure 10(b)) and contrast (Figure 10(c)).

Comparison with the NEMA NU 4-2008 image quality phantom concerned mainly the spatial resolution since for uniformity; there should not be any remarkable difference because the verification of this parameter was performed in the two phantoms in the same way, i.e. in a homogeneous region without any patterns. Thus, concerning spatial resolution, if the NEMA phantom permits to check the detectability of small hot lesions, the proposed phantom added to detectability the distinction of adjacent lesions which is the main principle of spatial resolution. Thus, looking at the test carried out on NEMA phantom (Figure 11(a)), we could find out that the resolution of the micro-SPECT unit is better than $1 \mathrm{~mm}$, but looking at the acquisition made with the proposed phantom (Figure 11(b)) we found out that the spatial resolution of this unit is about $1 \mathrm{~mm}$.

While the proposed phantom allows the evaluation of geometric distortions whether in micro-SPECT or microCT units, the NEMA phantom does not allow this operation. Moreover, the NEMA phantom is not flexible because it doesn't allow the introduction of other patterns while the considered phantom is extremely flexible because it allows the introduction and change of patterns inside. Thus with regard to the contrast measurement of 

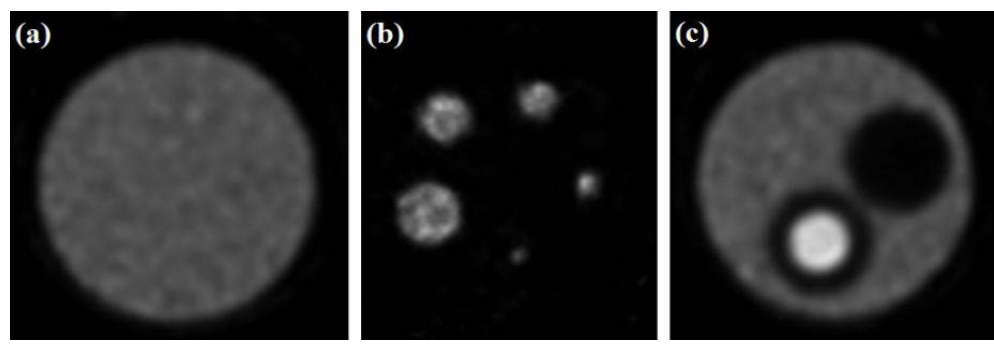

Figure 10. Acquisitions with NEMA NU 4-2008 image quality phantom ((a) Uniformity, (b) Spatial resolution, (c) Contrast).
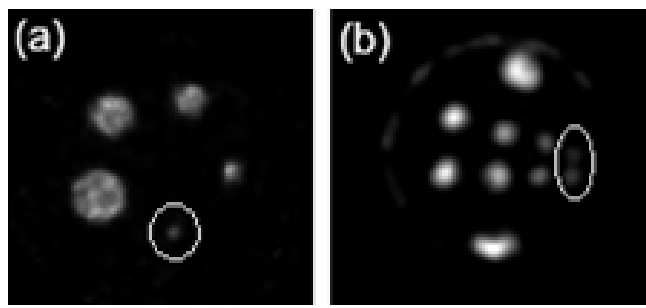

Figure 11. Spatial resolution ((a) NEMA NU 4-2008 phantom, (b) The phantom).

air and water in micro-PET units, it is very easy to realize this pattern and to insert it inside the phantom in case of quality control of micro-PET/CT systems.

\section{Conclusion}

This work offers to the practitioners a new tool to better achieve quality control protocols and obtain better quality parameter results. Indeed, the realized phantom presents very interesting qualities because it allows the measurement of whatever quality parameters for micro-SPECT/CT or micro-PET/CT as spatial resolution, spatial linearity, uniformity, slice thickness, Hounsfield unit linearity and diffusion profile. The proposed phantom is a first hybrid phantom for simultaneously quality control of micro-CT and micro-nuclear imaging systems. The comparison with the NEMA NU 4-2008 image quality phantom shows that the proposed phantom is more efficient regarding the evaluation of spatial resolution. The proposed phantom is also more flexible because it allows inserting patterns according to the device and objectives of quality control. It is easy to realize, very efficient and cheaper than other phantoms providing less opportunities in terms of quality control protocols.

\section{References}

[1] Glover, D.K., Kundu, B. and Schelbert, H.R. (2010) State-of-the-Art Instrumentation for PET and SPECT Imaging in Small Animals. In: Zaret, B.L. and Beller, G.A., Eds., Clinical Nuclear Cardiology: State of the Art and Future Directions, Mosby, Elsevier, Amsterdam, 163-179.

[2] Dillenseger, J.P., Guillaud, B., Goetz, C., Sayeh, A., Schimpf, R., Constantinesco, A. and Choquet, P. (2013) Coregistration of Data Sets from a Micro-SPECT/CT and a Preclinical 1.5T MRI. Nuclear Instruments and Methods in Physics Research A, 702, 144-147. http://dx.doi.org/10.1016/j.nima.2012.08.023

[3] Lina, K., Liu, H., Hsu, P., Chung, Y., Huang, W., Chen, J., Wey, S., Yen, T. and Hsiao, I. (2009) Quantitative MicroSPECT/CT for Detecting Focused Ultrasound-Induced Blood-Brain Barrier Opening in the Rat. Nuclear Medicine and Biology, 36, 853-861. http://dx.doi.org/10.1016/j.nucmedbio.2009.04.011

[4] Peremans, K., Vermeire, S., Dobbeleir, A., Gielen, I., Samoy, Y., Piron, K., Vandermeulen, E., Slegers, G., Van Bree, H., De Spiegeleer, B. and Dik, K. (2011) Recognition of Anatomical Predilection Sites in Canine Elbow Pathology on Bone Scans Using Micro-Single Photon Emission Tomography. The Veterinary Journal, 188, 64-72. http://dx.doi.org/10.1016/j.tvjl.2010.02.013

[5] Matthews, P.M., Coatney, R., Alsaid, H., Jucker, B., Ashworth, S., Parker, Ch. and Changani, K. (2013) Technologies: Preclinical Imaging for Drug Development. Drug Discovery Today: Technologies. Translational Pharmacology: From Animal to Man and Back, 10, 343-350. 
[6] Slavine, N.V. and Antich, P.P. (2008) Practical Method for Radioactivity Distribution Analysis in Small-Animal PET Cancer Studies. Applied Radiation and Isotopes, 66, 1861-1869. http://dx.doi.org/10.1016/j.apradiso.2008.06.008

[7] Moran, C.M., Pye, S.D., Ellis, W., Janeczko, A., Morris, K.D., Mcneilly, A.S. and Fraser, H.M. (2011) A Comparison of the Imaging Performance of High Resolution Ultrasound Scanners for Preclinical Imaging. Ultrasound in Medicine \& Biology, 37, 493-501. http://dx.doi.org/10.1016/j.ultrasmedbio.2010.11.010

[8] Jan, M., Ni, Y., Chen, K., Liang, H., Chuang, K. and Fu, Y. (2006) A Combined Micro-PET/CT Scanner for Small Animal Imaging. Nuclear Instruments and Methods in Physics Research A, 569, 314-318. http://dx.doi.org/10.1016/j.nima.2006.08.106

[9] Habraken, J.B.A., De Bruin, K., Shehata, M., Booij, J., Bennink, R., Van Eck Smit, B.L.F. and Sokole, E.B. (2001) Evaluation of High-Resolution Pinhole SPECT Using a Small Rotating Animal. The Journal of Nuclear Medicine, 42, 1863-1869.

[10] DeWerd, L.A. and Kissick, M. (2014) The Phantoms of Medical and Health Physics: Devices for Research and Development. Springer, Heidelberg. http://dx.doi.org/10.1007/978-1-4614-8304-5

[11] Busemann Sokole, E. (2003) IAEA Quality Control Atlas for Scintillation Camera Systems. IAEA Library Cataloguing in Publication Data.

[12] International Atomic Energy Agency (2014) PET/CT Atlas on Quality Control and Image Artefacts. IAEA Human Health Series, ISSN 2075-3772, No. 27.

[13] International Atomic Energy Agency (2012) Quality Assurance Programme for Computed Tomography: Diagnostic and Therapy Applications. IAEA Human Health Series, ISSN 2075-3772, No. 19.

[14] Cierniak, R. (2011) X-Ray Computed Tomography in Biomedical Engineering. Springer-Verlag London Limited, London. http://dx.doi.org/10.1007/978-0-85729-027-4

[15] Hsieh, H. and Hsiao, I. (2010) Image Reconstructions from Limit Views and Angle Coverage Data for a Stationary Multi-Pinhole SPECT System. Tsinghua Scienece and Technology, 15, 44-49.

[16] Kupinski, M.A. and Barrett, H.H. (2005) Small-Animal SPECT Imaging. Springer Science+Business Media, Inc., Heidelberg.

[17] Seret, A. (2011) NEMA NU1-2001 Performance Tests of four Philips Brightview Cameras. Nuclear Instruments and Methods in Physics Research Section A: Accelerators, Spectrometers, Detectors and Associated Equipment, 648, S89S92. http://dx.doi.org/10.1016/j.nima.2010.12.104

[18] Panetta, D., Belcari, N., Del Guerra, A., Bartolomei, A. and Salvadori, P.A. (2012) Analysis of Image Sharpness Reproducibility on a Novel Engineered Micro-CT Scanner with Variable Geometry and Embedded Recalibration Software. Physica Medica, 28, 166-173. http://dx.doi.org/10.1016/j.ejmp.2011.03.006

[19] Disselhorst, J.A., Brom, M., Laverman, P., Slump, C.H., Boerman, O.C., Oyen, W.J.G., Gotthardt, M. and Visser, E.P. (2010) Image-Quality Assessment for Several Positron Emitters Using the NEMA NU 4-2008 Standards in the Siemens Inveon Small-Animal PET Scanner. The Journal of Nuclear Medicine, 51, 610-617. http://dx.doi.org/10.2967/jnumed.109.068858

[20] Anizan, N., Carlier, T., Hindorf, C., Barbet, J. and Bardiès, M. (2012) Acquisition Setting Optimization and Quantitative Imaging for ${ }^{124}$ I Studies with the Inveon MicroPET-CT System. EJNMMI Research, 2, 1-9.

http://dx.doi.org/10.1186/2191-219X-2-7

http://www.ejnmmires.com/content/2/1/7 
Scientific Research Publishing (SCIRP) is one of the largest Open Access journal publishers. It is currently publishing more than 200 open access, online, peer-reviewed journals covering a wide range of academic disciplines. SCIRP serves the worldwide academic communities and contributes to the progress and application of science with its publication.

Other selected journals from SCIRP are listed as below. Submit your manuscript to us via either submit@scirp.org or Online Submission Portal.
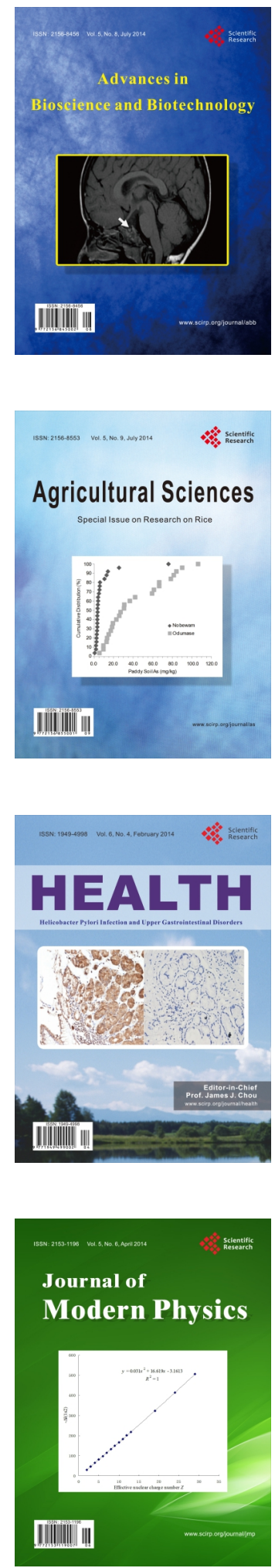
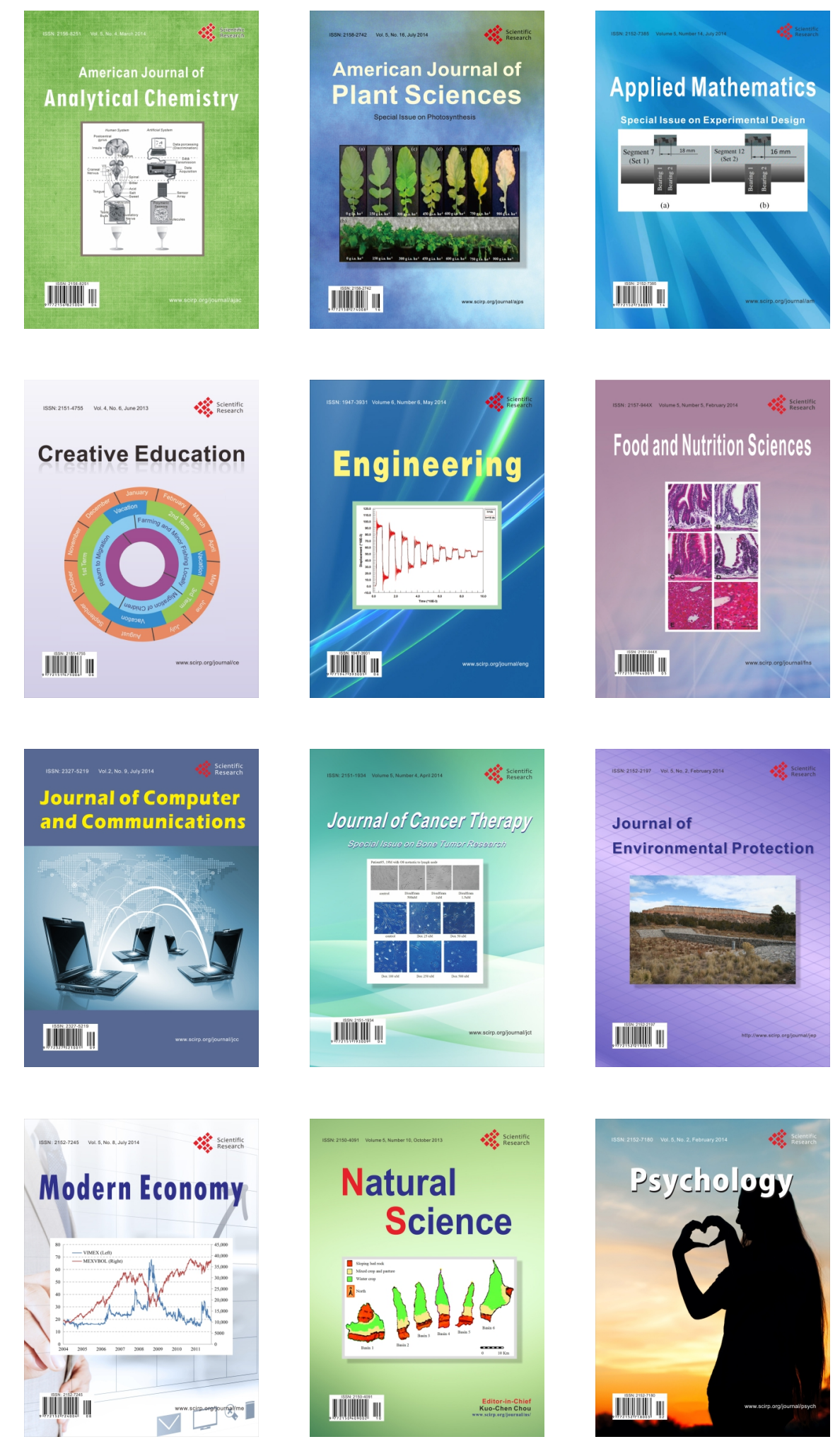